\title{
Impacto da doença periodontal na qualidade de vida: uma revisão integrativa
}

\author{
Impact of periodontal disease on quality of life: an integrative review \\ Impacto de la enfermedad periodontal en la calidad de vida: una revisión integrativa
}

\author{
Mauro Bezerra do Nascimento Júnior \\ ORCID: https://orcid.org/0000-0002-3116-1013 \\ Universidade do Estado do Rio Grande do Norte, Brasil \\ E-mail: maurojunior@alu.uern.br \\ Fernando José de Oliveira Nóbrega \\ ORCID: https://orcid.org/0000-0002-0121-0996 \\ Universidade do Estado do Rio Grande do Norte, Brasil \\ E-mail: nobrega.fernando@hotmail.com \\ Eloísa Cesário Fernandes \\ ORCID: https://orcid.org/0000-0002-9330-1245 \\ Universidade do Estado do Rio Grande do Norte, Brasil \\ E-mail: eloisa_cesario@hotmail.com \\ Micássio Fernandes de Andrade \\ ORCID: https://orcid.org/0000-0003-2836-9080 \\ Universidade do Estado do Rio Grande do Norte, Brasil \\ E-mail: micassiofernandes@gmail.com \\ Camila Cristine Araújo de Oliveira \\ ORCID: https://orcid.org/0000-0001-5376-3173 \\ Universidade do Estado do Rio Grande do Norte, Brasil \\ E-mail: camilaodonto06@gmail.com \\ Antônio Eliece Fernandes Filho \\ ORCID: https://orcid.org/0000-0002-5865-6331 \\ Faculdade de Medicina Nova Esperança, Brasil \\ E-mail: eliecefarma1@gmail.com \\ Patrícia Bittencourt Dutra dos Santos \\ ORCID: https://orcid.org/0000-0003-3308-6376 \\ Universidade do Estado do Rio Grande do Norte, Brasil \\ E-mail: pati_bittencourt@hotmail.com
}

\begin{abstract}
Resumo
Objetivo: Realizar uma revisão de literatura, com busca sistemática, observando as evidências quanto ao impacto da doença periodontal na qualidade de vida. Metodologia: A pesquisa foi feita nas bases de dados Medline/Pubmed, SciELO, LILACS, Web of Science e Scopus, nas quais utilizou-se os descritores "Periodontal Diseases" e "Quality of Life". Foram incluídos artigos publicados em português, inglês e espanhol que retrataram a temática no período compreendido entre 2015-2020. Excluiu-se os artigos duplicados e todos os estudos de revisão. Inicialmente, identificou-se 1435 artigos, restando 36 artigos após a observação dos critérios de elegibilidade. Resultados: A doença periodontal causa um impacto negativo na qualidade de vida relacionada à saúde oral, visto que, do total de artigos $(\mathrm{n}=36)$, trinta evidenciaram essa afirmativa, e quanto mais severa é a destruição periodontal, pior é a qualidade de vida $(n=11)$. Entretanto, alguns estudos $(n=4)$ não encontraram relações significativas entre a doença e a qualidade de vida ou obtiveram baixo impacto. Além disso, há resultados com alto impacto na qualidade de vida nos seguintes grupos: fumantes, ausência de seguro de saúde, obesos e diabéticos, grávidas, ansiosos, idosos, pacientes com doença renal terminal, cárie, hipertensão arterial. Conclusão: As doenças periodontais interferem negativamente na qualidade de vida das pessoas afetadas e há evidências de quanto maior a severidade da doença, maior é o seu impacto na qualidade de vida. Contudo, são necessários estudos clínicos randomizados e estudos do tipo longitudinal para fortalecer esses achados.
\end{abstract}

Palavras-chave: Doenças periodontais; Qualidade de vida; Saúde bucal; Periodontia.

\begin{abstract}
Objective: To carry out a literature review, with a systematic search, observing the evidence regarding the impact of periodontal disease on quality of life. Methodology: The research was carried out in the Medline / Pubmed, SciELO, LILACS, Web of Science and Scopus databases, in which the descriptors "Periodontal Diseases" and "Quality of Life" were used. Articles published in Portuguese, English and Spanish that portrayed the theme in the period between $2015-$ 2020 were included. Duplicate articles and all review studies were excluded. Initially, 1435 articles were identified, leaving 36 articles after observing the eligibility criteria. Results: Periodontal disease has a negative impact on quality
\end{abstract}


of life related to oral health, since, of the total articles $(n=36)$, thirty evidenced this statement, and the more severe the periodontal destruction, the worse the quality of life. life $(n=11)$. However, some studies $(n=4)$ have found no significant relationship between the disease and quality of life or have obtained low impact. In addition, there are results with a high impact on quality of life in the following groups: smokers, absence of health insurance, obese and diabetics, pregnant women, anxious people, elderly people, patients with terminal kidney disease, caries, high blood pressure. Conclusion: Periodontal diseases negatively affect the quality of life of affected people and there is evidence that the greater the severity of the disease, the greater its impact on quality of life. However, randomized clinical studies and longitudinal studies are needed to strengthen these findings.

Keywords: Periodontal diseases; Quality of life; Oral health; Periodontics.

\section{Resumen}

Objetivo: Realizar una revisión de la literatura, con una búsqueda sistemática, observando la evidencia sobre el impacto de la enfermedad periodontal en la calidad de vida. Metodología: La investigación se realizó en las bases de datos Medline / Pubmed, SciELO, LILACS, Web of Science y Scopus, en las que se utilizaron los descriptores "Enfermedades periodontales" y "Calidad de vida". Se incluyeron artículos publicados en portugués, inglés y español que retrataron el tema en el período 2015-2020. Se excluyeron los artículos duplicados y todos los estudios de revisión. Inicialmente se identificaron 1435 artículos, quedando 36 artículos después de observar los criterios de elegibilidad. Resultados: La enfermedad periodontal tiene un impacto negativo en la calidad de vida relacionada con la salud bucal, ya que, del total de artículos $(\mathrm{n}=36)$, treinta evidenciaron esta afirmación, y cuanto más severa es la destrucción periodontal, peor es la calidad de vida. $(n=11)$. Sin embargo, algunos estudios $(n=4)$ no han encontrado una relación significativa entre la enfermedad y la calidad de vida o han obtenido un impacto bajo. Además, existen resultados con alto impacto en la calidad de vida en los siguientes grupos: fumadores, ausencia de seguro médico, obesos y diabéticos, mujeres embarazadas, personas ansiosas, ancianos, pacientes con enfermedad renal terminal, caries, hipertensión arterial. Conclusión: Las enfermedades periodontales afectan negativamente la calidad de vida de las personas afectadas y existe evidencia de que cuanto mayor es la gravedad de la enfermedad, mayor es su impacto en la calidad de vida. Sin embargo, se necesitan estudios clínicos aleatorizados y estudios longitudinales para fortalecer estos hallazgos.

Palabras clave: Enfermedades periodontales; Calidad de vida; Salud bucal; Periodoncia.

\section{Introdução}

Desde a filosofia grega e, inclusive, nas reflexões de Aristóteles já havia a identificação do conceito de qualidade de vida (Mendez \& Gomes, 2013). Ao longo do tempo, esse conceito foi passando por várias modificações que refletem transformações no contexto médico e científico e sua relação com a história, a cultura e a política (Lopes, Gusmão, Alves, \& Cimões, 2011). Nessa perspectiva, em 1995 o grupo The World Health Organization Quality of Life (WHOQOL) da Organização Mundial da Saúde definiu qualidade de vida como "a percepção do indivíduo da sua posição na vida, de acordo com o contexto cultural e os valores com os quais convive, em relação aos seus objetivos, expectativas, padrões e preocupações"("The World Health Organization Quality of Life assessment (WHOQOL): position paper from the World Health Organization," 1995). Dessa forma, esse termo engloba fatores como saúde física, estado psicológico, nível de dependência, relações sociais, crença e as relações dos indivíduos com questões do ambiente em que se encontram (Mendez \& Gomes, 2013).

Segundo o relatório da organização mundial da saúde, as doenças bucais implicam em um enorme impacto na qualidade de vida englobando todas as idades haja vista que diminui a autoestima, a capacidade de se alimentar, nutrição e saúde, como também causa dor, ansiedade e privações sociais (Mendez \& Gomes, 2013). Dessa forma, atualmente, a odontologia procura não focar somente no problema odontológico e sim observar as particularidades de cada indivíduo que pode afetar nas questões sistêmicas do organismo (Kreve \& Anzolin, 2016).

A doença periodontal tem como fator etiológico primário o biofilme subgengival, cuja composição é definida principalmente por bactérias gram-negativas que causam um conjunto de condições inflamatórias. Isso traz a destruição do tecido de suporte do dente, ocorrendo a perda progressiva de tecido conjuntivo. No entanto, apenas a presença desses microrganismos não é capaz de promover o início ou a intensidade da doença (Dantas, Martins, Dantas, \& Gnoatto, 2016). Diante disso, dentro das doenças bucais, a periodontal é uma das mais recorrentes no mundo, considerada um problema relevante de saúde pública, além da cárie dentária, e é a causa mais comum de perda dentária em adultos de países desenvolvidos e em desenvolvimento (Neves et al., 2013). Isso implica em impactos estéticos, funcionais, psicológicos e sociais, se tornando um verdadeiro problema 
de saúde pública pelo fato de atingir a autoestima do paciente (Kreve \& Anzolin, 2016).

Dessa forma, viu-se a necessidade do uso de indicadores de qualidade de vida através de uma avaliação subjetiva do impacto que as diversas condições orais trazem para o paciente. Diante da relevância do tema, o objetivo principal deste trabalho foi realizar uma revisão da literatura para encontrar evidências quanto ao impacto da doença periodontal na qualidade de vida.

\section{Metodologia}

Trata-se de uma revisão integrativa com coleta de dados realizada através de levantamentos bibliográficos. Inicialmente, foi adotada a seguinte questão norteadora: A doença periodontal tem impacto na qualidade de vida dos pacientes?

Para responder a essa pergunta, foram realizadas revisões da literatura, buscando reunir e sintetizar os resultados encontrados nos estudos primários que analisaram a relação entre a doença periodontal e a qualidade de vida. Para isso, foram utilizados artigos científicos coletados nas bases de dados do Medline/Pubmed, SciELO, LILACS, Web of Science e Scopus. A busca ocorreu entre o período de abril e maio de 2020.

Foram utilizados para busca dos artigos, de acordo com o Descritor em Ciências da Saúde (DeCS) e o sistema Medical Subject Headings (MeSH), os seguintes descritores: "Periodontal Diseases" e "Quality of Life". A estratégia de busca se deu através da combinação dos dois descritores com o operador booleano AND em todas as bases de dados, desta forma, ampliouse a amostragem da pesquisa, minimizando possíveis vieses na busca dos artigos. Além disso, foi utilizado filtro de data de publicação para obter os artigos dos últimos 5 anos (2015-2020).

Os critérios de inclusão definidos para a seleção dos artigos foram: artigos publicados em português, inglês e espanhol que retrata a temática no período compreendido entre 2015-2020, possuir resumo disponível nas bases de dados selecionadas e artigos na íntegra com acesso aberto via Portal de Periódicos Capes. Foram excluídos os artigos duplicados e todos os estudos de revisão.

As etapas de extração e análise dos resultados dos estudos primários foram realizadas por um único revisor e o processo de seleção seguiu a ordem: etapa de seleção pela leitura do título; etapa de seleção pela leitura dos resumos e etapa de seleção pela leitura na íntegra dos artigos.

Após a consulta nas bases de dados, foram identificados 1435 artigos potencialmente elegíveis, deste total, foram excluídos 317 artigos, após a análise de duplicata. Na etapa de seleção pelo título foram removidos 845 artigos, restando 273 artigos. Essa etapa foi seguida pela leitura dos resumos de cada publicação, sendo removidos 216 estudos, totalizando 57 artigos para a análise de leitura na íntegra. Após esta etapa, 20 artigos foram excluídos por não cumprirem os critérios de elegibilidade, totalizando um restante de 37 artigos para serem incluídos nesta revisão integrativa (Figura 1). 
Figura 1: Fluxograma de seleção dos artigos para a revisão integrativa.

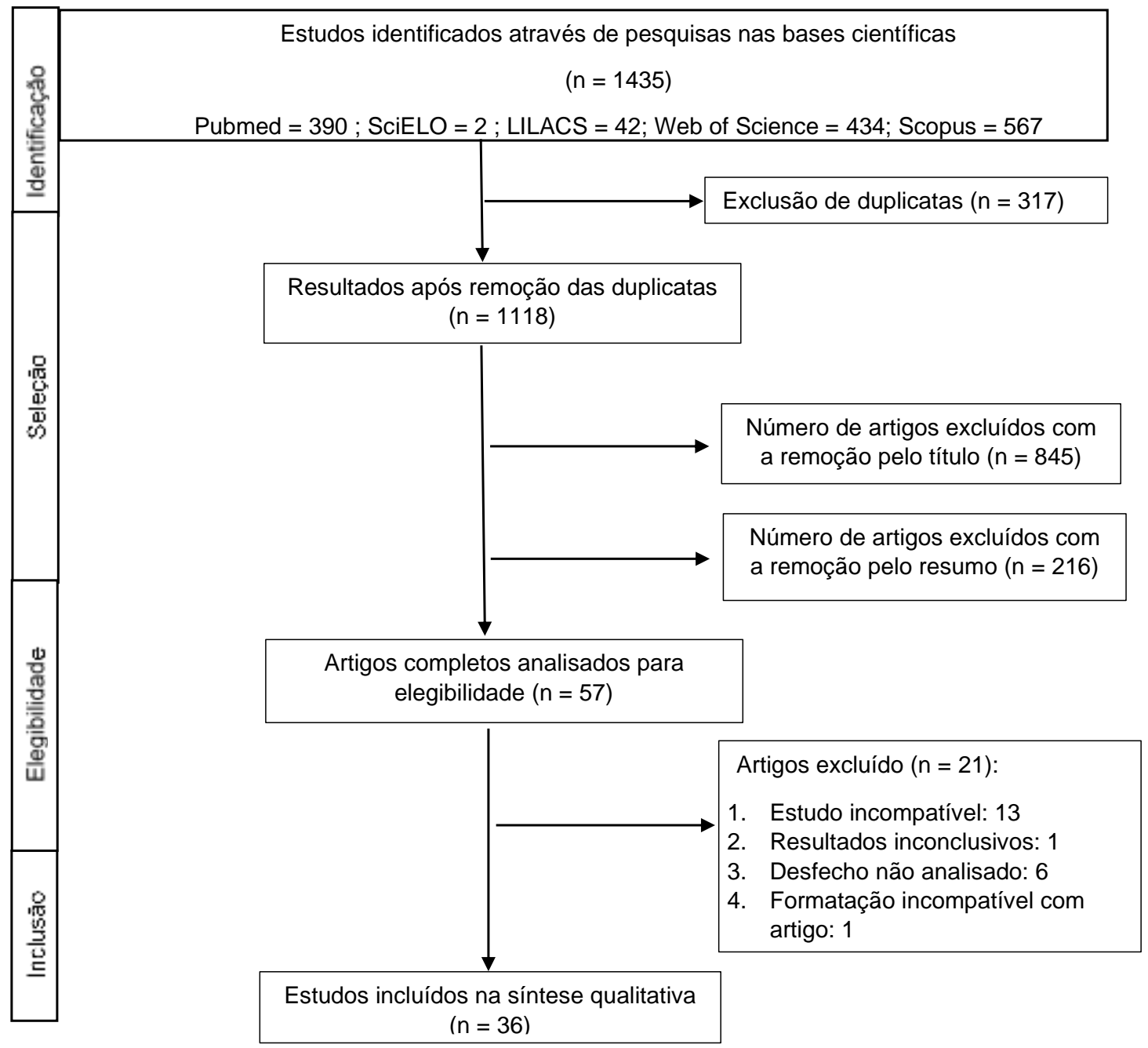

Fonte: Autores.

Posteriormente à seleção dos artigos, foi feito um resumo organizado de seus conteúdos. O processo de extração de dados dos artigos que passaram por triagem foi guiado por uma ficha de análise padrão, elaborada em caráter prévio e empregada na avaliação dos estudos recrutados em todas as estratégias de busca supracitadas.

\section{Resultados}

A Tabela 1 apresenta a síntese dos artigos que atenderam aos critérios de inclusão previamente estabelecidos e foram incluídos nesta revisão integrativa. Os seguintes dados foram extraídos dos estudos selecionados: Autor, ano da publicação, tamanho e tipo da amostra, intervenção estudada, resultados e conclusões. As pesquisas foram realizadas no Brasil (n=8), Índia $(n=5)$, Turquia $(n=4)$, Reino Unido $(n=3)$, China $(n=3)$, Israel $(n=2)$, Arábia Saudita $(n=2)$, Irã $(n=1)$, Nepal $(n=1)$, Noruega $(\mathrm{n}=1)$, Suécia $(\mathrm{n}=1)$, Bulgária $(\mathrm{n}=1)$, Alemanha $(\mathrm{n}=1)$, Sri Lanka $(\mathrm{n}=1)$, Estados Unidos $(\mathrm{n}=1)$, Malásia $(\mathrm{n}=1)$. 
Tabela 1. Apresentação da síntese de artigos incluídos na revisão integrativa.

\begin{tabular}{ccc}
\hline Autor e ano/ & Intervenção estudada & Resultados \\
Tamanho e tipo da & & \\
amostra & &
\end{tabular}

\begin{tabular}{|c|c|}
\hline & $\begin{array}{l}\text { Avaliaram a influência da gravidade } \\
\text { da doença periodontal na qualidade de }\end{array}$ \\
\hline $\begin{array}{l}\text { Al-Kattan, R. et al. } \\
2019 /\end{array}$ & $\begin{array}{l}\text { vida }(\mathrm{QV}) \text { com o uso de uma versão } \\
\text { curta do Perfil de Impacto na Saúde }\end{array}$ \\
\hline $\begin{array}{l}\text { Conveniência/ } 25 \\
(21-80 \text { anos })\end{array}$ & $\begin{array}{l}\text { Oral (OHIP-14) em pacientes com } \\
\text { doença periodontal. }\end{array}$ \\
\hline
\end{tabular}

\begin{tabular}{ll}
\hline Andrade, A. L.et al. & $\begin{array}{l}\text { Verificaram se há associação entre } \\
\text { autopercepção de qualidade de vida e } \\
\text { doença periodontal. Os instrumentos }\end{array}$ \\
$\begin{array}{l}\text { observacional } \\
\text { transversal }\end{array}$ & e $\begin{array}{l}\text { utilizados foram o (OHIP-14) e exame } \\
\text { clínico periodontal. }\end{array}$
\end{tabular}

analítico / $200(13$ a

21 anos)
O impacto da doença periodontal na QV foi estatisticamente significativo para dor física e incapacidade psicológica. A gravidade da doença periodontal não mostrou impacto negativo na limitação funcional.
A destruição periodontal tem um impacto negativo na QV. O uso desse índice, tem implicações significativas na periodontia, incluindo o planejamento do tratamento e a avaliação dos resultados do tratamento.
Não foi possível identificar a associação entre a doença periodontal e o impacto negativo na qualidade de vida dos adolescentes.
Sugere-se que a ausência do impacto negativo seja devido à uma fase inicial da periodontite. Os adolescentes acometidos pela doença não conseguem identificar as consequências da doença.

\section{Ansari Moghadam, Determinaram a relação entre S. et al. 2015 / condição periodontal e qualidade de amostragem aleatória. / 700 ( com mais de 35 anos) vida. Para avaliar a saúde geral, foi utilizado o questionário de qualidade de vida da OMS (WHOQOL-BREF).}

\section{El Tantawi, M.}

AlAgl, A. 2017 /estudo transversal/ 1098 (maiores de 18 anos)

\section{Compararam a necessidade de} cuidados periodontais e seu impacto na vida diária entre adultos com deficiência e saudáveis.

\section{Com a deterioração da condição periodontal das pessoas, sua qualidade de vida também diminuía, bem como o escore total relacionados a saúde.}

As pessoas saudáveis e com deficiência tiveram grande necessidade de cuidados periodontais. Os fumantes tinham uma maior probabilidade e os com seguro tinham uma menor probabilidade de necessidade de cuidados periodontais.
Indica uma diminuição da qualidade de vida geral em pacientes com periodontite. Os autores sugerem estudos com amostras maiores, como estudos de coorte.

A maioria dos adultos
precisaram de cuidados periodontais, e as pessoas com deficiência experimentaram um maior impacto na qualidade de vida. Fumantes e pessoas sem seguro eram mais propensas a precisar de cuidados periodontais impactando a vida diária.

\begin{abstract}
Eltas, A. et al. 2016 / Avaliaram a associação da qualidade delineamento de vida relacionada à saúde bucal transversal/ 404 (OHRQoL) com parâmetros (maiores de 20 anos) periodontais e necessidades de tratamento. O impacto da saúde bucal na QV foi avaliado pelo OHIP-14.
\end{abstract}

Os escores em indivíduos com gengivite e periodontite foram maiores do que em indivíduos saudáveis. Os efeitos adversos na qualidade de vida percebidos pelos pacientes foram semelhantes nos indivíduos com gengivite e periodontite.

A periodontite esteve associada ao Diabetes Mellitus. O impacto bucal na qualidade de vida foi baixo e a SV não diferiu entre os grupos. A qualidade de vida foi correlacionada com idade e dentes perdidos.
As doenças periodontais podem qualidade de vida. Estudos longitudinais são necessários para esclarecer a relação entre o status periodontal e a qualidade de vida.

A ocorrência de periodontite no período pré-operatório foi maior nos pacientes obesos diabéticos. O impacto bucal na qualidade de vida foi baixo entre os grupos. afetar significativamente a prospectivo/ 150 e pacientes obesos diabéticos e não diabéticos submetidos à cirurgia bariátrica. Aplicou-se OHIP-14 e Escala de Satisfação com a Vida (SV), além do registro das condições socioeconômicas, hábitos e história médica. 
Fuller, J. et al. 2020
/desenho de casocontrole /

461 (média de 39 anos)
Avaliaram a associação entre qualidade de vida e presença de diferentes gravidades e formas de periodontite (agressiva e crônica), em comparação com um grupo controle livre de doença. A qualidade de vida relacionada à saúde bucal foi avaliada usando o questionário OHIP-14

\section{Foram encontradas diferenças} significativas entre os grupos de periodontite e controles saudáveis para os escores de resultados e em todos os domínios do OHIP-14. Não foram identificadas diferenças significativas entre o periodontite agressiva e crônica.
Geevarghese, A. et al. 2017 / estudo transversal / 300 (18 a 35 anos)
Avaliaram a diferença na qualidade de vida relacionada à saúde bucal (QVRS) e no estado de saúde bucal entre gestantes e não gestantes. Os dados foram coletados através de exame oral e questionário de perfil de impacto à saúde bucal-49 (OHIP-49).
As médias da doença periodontal e a perda de inserção clínica em gestantes foram significativamente maiores que as das não gestantes. A periodontite e o estado gravídico tiveram uma relação linear positiva com os escores do OHIP49.

Pacientes com periodontite têm pior qualidade de vida que indivíduos periodicamente saudáveis, sendo as diferenças clinicamente significativas. Pacientes com periodontite agressiva relataram pior qualidade de vida em relação aos pacientes com periodontite crônica, mas essas diferenças moderadas e significativas foram explicadas pelo processo de ajuste.

A saúde periodontal e a QVRS das mulheres grávidas foram piores que as não gestantes. Destaca-se a necessidade de promoção efetiva dos cuidados com a saúde bucal por meio de programas focados na população grávida.

\begin{tabular}{|c|c|}
\hline Goel, K. & $\begin{array}{l}\text { Avaliaram o impacto das doenças } \\
\text { periodontais crônicas } \\
\text { (DP) }\end{array}$ \\
\hline $\begin{array}{l}\text { Baral, D. } 2017 \text { / } \\
\text { estudo transversal } \\
\text { comparativo/ }\end{array}$ & $\begin{array}{l}\text { compararam as fases da terapia } \\
\text { periodontal não cirúrgica (NSPT) na } \\
\text { qualidade de vida relacionada à saúde }\end{array}$ \\
\hline 49 (17-60 anos) & $\begin{array}{l}\text { bucal (OHRQoL. Foi utilizado o } \\
\text { OHIP-14 para avaliar o impacto da } \\
\text { condição periodontal. }\end{array}$ \\
\hline
\end{tabular}

A pontuação total OHIP-14 para doença periodontal reduziu de 7 para 3 após o tratamento não cirúrgico. Ambos os grupos apresentaram uma melhora significativa na OHRQoL. O grupo periodontite mostrou uma mediana aumentada de $52 \%$ em comparação com o grupo gengivite com $27 \%$. Foi observado o impacto na dor orofacial, na aparência orofacial e nas dimensões psicossociais.

\section{As doenças periodontais afetam negativamente a qualidade de vida em muitos aspectos, especialmente naqueles indivíduos que sofrem de periodontite crônica generalizada. A terapia periodontal não cirúrgica tem um papel a desempenhar para melhorar o impacto, com mudanças significativas \\ observada para descamação da superfície da raiz e debridamento radicular procedimento.}

À medida que a gravidade da doença periodontal aumenta, observa-se pior qualidade de vida relacionada à saúde bucal. A avaliação da qualidade de vida relacionada à saúde bucal pode desempenhar um papel importante na prática clínica em relação à identificação das condições de saúde bucal dos pacientes e das necessidades de tratamento e à melhoria da comunicação do paciente.

A periodontite crônica foi associada com pior QVRS em chineses adultos. Além disso, o comprometimento da QVRS mostrou correlação significativa

$\begin{array}{llll}\text { He, S. et al. 2018 / } & \text { Exploraram a associação entre } & \text { Os participantes com periodontite } \\ \text { Transversal com } & \text { periodontite crônica e qualidade de } & \text { crônica grave e moderada apresentaram } \\ \text { amostragem } & & \text { vida relacionada à saúde bucal } & 1,63 \text { e } 1,42 \text { vezes mais chances de } \\ \text { aleatória } & \text { (QVRS), a versão em chinês do perfil } & \text { apresentarem escores OHIP-14 acima da } \\ & \text { de impacto na saúde oral em formato } & \text { mediana em comparação aos } \\ & & \text { participantes sem periodontite crônica. }\end{array}$


estratificada / 480 curto (OHIP-14) foi usada para Os participantes com periodontite leve (35 a 74 anos) avaliar a QVRS. não tiveram escores maiores do OHIP14 do que os participantes sem periodontite crônica. com a gravidade da periodontite crônica.

\begin{abstract}
Holde, G. E. et al. Examinaram as relações diretas e 2018 / Transversal/ indiretas entre características da 1.819 (20 a 79 anos) população, comportamentos de saúde bucal, periodontite e impactos na saúde bucal, a condição socioeconômico, o senso de coerência, a ansiedade dental, a necessidade percebida de tratamento, os comportamentos de saúde bucal e o perfil de impacto na saúde bucal (OHIP-14) foram coletados por meio de questionário.
\end{abstract}

\section{A periodontite foi associada ao aumento dos impactos na saúde bucal. Um maior número de serviços odontológicos esteve associado a menos impactos orais. Não houve associação entre uso de serviços odontológicos e impactos na saúde bucal. \\ Em pacientes não diabéticos, houve escores mais altos em para gengivite, embora tal efeito não tenha sido observado nos pacientes com diabetes. Nos pacientes não diabéticos com periodontite, foram observadas reduções estatisticamente no desconforto psicológico e incapacidade psicológica após o tratamento periodontal.}

\begin{tabular}{|c|c|}
\hline Karaaslan, F. & Investigaram a associação da \\
\hline $\begin{array}{l}\text { Dikilitas, A. } 2019 \text { / } \\
\text { Tranversal/ }\end{array}$ & $\begin{array}{l}\text { efeito da periodontite na qualidade de } \\
\text { vida, O estudo consistiu em exame }\end{array}$ \\
\hline 99 (18 a 40 anos) & $\begin{array}{l}\text { clínico e questionário para qualidade } \\
\text { de vida (OHIP-14) e Índice de } \\
\text { qualidade do Sono de Pittsburgh } \\
\text { (PSQI) }\end{array}$ \\
\hline
\end{tabular}

A média do escore global do OHIP-14 foi de $13,43+/-6,23$ e a pontuação global média do PSQI foi de $6,57+/-$ 3,53 .
Fatores socioeconômicos e tabagismo foram os principais preditores de periodontite. Hábitos regulares de visita odontológica, não reduziram a probabilidade de periodontite. Um maior escore para periodontite foi associado ao aumento dos impactos na saúde bucal.. Não houve associação entre uso de serviços odontológicos e impactos na saúde bucal.

A gengivite e a periodontite estão associados à redução da QVRS em comparação à saúde periodontal em pacientes não diabéticos, com melhorias após o tratamento da periodontite.
O estágio de periodontite foi associado à curta duração do sono, baixa qualidade do sono e baixa qualidade de vida relacionada à saúde bucal.

\begin{abstract}
Kato, T. et al. 2018 / Avaliaram as associações entre o Derivado de dois estágio de doença periodontal e estudos de coorte/ número de dentes na qualidade de vida relacionada à saúde bucal em 804 ( media 70 anos) idosos. Foram realizados exames dentários e o questionário OHIP-14.
\end{abstract}

Nos participantes com 1 a 10 dentes restantes, o escore do OHIP-14 aumentou significativamente indicando uma qualidade de vida ruim.
A periodontite não mostrou associação com uma qualidade de vida ruim, no entanto, foi encontrada uma associação significativa entre o número de dentes e uma qualidade de vida ruim.

\footnotetext{
Levin, L. et at. 2018 Examinaram se a ansiedade dental e a / transversal qualidade de vida relacionada à saúde comparativo/

148 (18 a 65 anos)
}

\footnotetext{
Pacientes com periodontite crônica exibiram piores escores OHIP-14 e piores escores nos seguintes domínios individuais: limitação funcional, incapacidade física, incapacidade psicológica, incapacidade social e desvantagem.
Comparados aos indivíduos do grupo controle, os pacientes com periodontite crônica apresentaram maiores níveis de ansiedade dental e pior considerar a ansiedade dental e a avaliação da qualidade de vida como um componente integral da avaliação de pacientes com periodontite crônica. qualidade de vida. É importante
} 
Levin, L. et al. 2018 Mediram os níveis de ansiedade / Caso controle (não dental e as qualidades de vida probabilístico) / relacionadas à saúde bucal (QVRS) em pacientes com periodontite agressiva (PA). Foram utilizados Escala de Ansiedade Odontológica (DAS) de Corah e Perfil de Impacto na Saúde Oral-14 (OHIP-14).
Comparados ao grupo controle, os pacientes com Periodontite agressiva generalizada exibiram piores escores no OHIP-14 global e em todos os domínios individuais do OHIP-14. Entre os pacientes com PA e o grupo controle, o domínio da dor física foi o maior impacto registrado, enquanto o menor impacto foi registrado no domínio da limitação funcional.

A doença periodontal apresentou menor probabilidade de uma melhor qualidade de vida relacionada à saúde bucal. Análises do mediador mostraram que a capacidade de mastigação era um mediador da associação entre QV relacionada à saúde bucal. A doença periodontal foi um fator independente para a $\mathrm{QV}$ relacionada à saúde bucal.

As médias globais da pontuação do OHIP-14 foram 16,5 para o periodontite agressiva generalizada e 17,5 para o periodontite crônica generalizada.

Pacientes com Periodontite Agressiva Generalizada foram associados com maiores níveis de ansiedade dental e pior QVRS. A auto percepção da ansiedade dentária e da QVRS deve ser considerada um elemento integrante do processo rotineiro de diagnóstico das doenças periodontais.

A doença periodontal é um fator de risco independente diretamente relacionado à $\mathrm{QV}$ relacionada à saúde bucal, enquanto a capacidade de mastigação é um mediador entre a QV e o uso de prótese removível.

Pacientes com diferentes formas de periodontite têm impacto na qualidade de vida dos pacientes quando medidos com o OHIP14. Pacientes com periodontite agressiva generalizada e periodontite crônica generalizada apresentaram pior qualidade de vida relacionada a saúde.

\section{Lu, H. X. et al. 2015 \\ / Estudo transversal/ \\ 512 (idade média 27,3 anos)}

Descreveram a qualidade de vida relacionada a saúde (QVRS) de mulheres grávidas em Xangai, na China, e investigaram as relações entre as condições periodontais e a QVRS de mulheres grávidas. A OHRQoL determinada pelo OHIP-14, (versão chinesa).
As condições periodontais não foram significativamente associadas a três formatos de pontuação de QVRS (gravidade, extensão e prevalência do impacto). A perda dentária foi significativa na extensão dos impactos negativos.
Gestantes com diferentes trimestres apresentaram impacto semelhante da doença bucal em sua QVRS. O estado de saúde periodontal não afeta a qualidade de vida relacionada a saúde bucal.

\begin{abstract}
Maia, C. D. R. et al. Avaliaram o impacto da cárie dentária 2018 / desenho e da doença periodontal na qualidade transversal/ 564 (15 de vida de adolescentes e adultos a 25 anos)
\end{abstract}

A presença de bolsas periodontais estava significativamente relacionada ao OHIP.
A cárie dentária e a doença periodontal afetam negativamente a qualidade de vida relacionada à saúde bucal. No entanto, essas doenças parecem afetar mais significativamente os indivíduos de comunidades remotas.

\begin{abstract}
Marya, C. et al. 2020
/ estudo transversal/

1.200 (acima de 60 anos)
\end{abstract}

A associação entre gênero do estado periodontal e a QVRS revelou uma associação significativa com os dentes móveis. Não foi observada relação entre QVRS, sangramento gengival, bolsa periodontal e perda de inserção.
Não foram observadas diferenças significativas na condição periodontal entre homens e mulheres.

Masood, M. et al. Investigaram, o impacto da doença
$2019 /$ Bolsa periodontal foi associado a Para


secundária de cortes qualidade de vida relacionada à saúde transversais/

$6378(16-65$ anos) bucal (OHRQoL) de adultos do Reino Unido, para isso, foi utilizado o questionário OHIP-14.

\begin{abstract}
Meusel, D. R. et al. Examinaram o impacto da gravidade 2015 / Transversal da doença periodontal na qualidade de (Recrutamento)/ vida de adultos com periodontite $100(30-58$ anos $)$ crônica, utilizando o questionário OHIP-14Br
\end{abstract}

Presença de bolsa periodontal $>3.5 \mathrm{~mm}$ população do Reino Unido, a tiveram uma prevalência doença periodontal estava significativamente maior de limitação significativamente associada as funcional, dor física e incapacidade variáveis da OHRQoL. social.

O escore OHIP-14Br foi maior no G2 (periodontite crônica grave) do que no G1 (Periodontite leve / moderado).

A gravidade da doença periodontal foi inversamente associada à qualidade de vida em adultos brasileiros.

\section{Musurlieva, N. \\ Stoykova, M. 2015 / \\ Estudo de corte \\ transversal/ \\ 228 (média de 50 anos)}

Examinaram uma ferramenta auto desenvolvida para avaliar o impacto da periodontite crônica na qualidade de vida $(\mathrm{QV})$. 2017 / Casocontrole (Recrutamento)/ impacto na qualidade de vida dos diabéticos tipo II. Foi utilizado o questionário OHIP-14.
Nayak, S. U. et al.

\begin{abstract}
Os aspectos negativos foram mais prevalentes em indivíduos com periodontite crônica grave. O domínio que mais influenciou a periodontite foi à saúde geral, seguida pela autoestima e aparência, e a função menos afetada foi a fala.
\end{abstract}

O escore de necessidade de tratamento periodontal foi estatisticamente significante quando comparado aos grupos controle e teste.

\begin{abstract}
A QV da maioria dos pacientes com periodontite crônica grave foi agravada. A ferramenta pode ser utilizada com sucesso na prática, o que ajudará os dentistas a avaliar facilmente a QV dos pacientes com periodontite.
\end{abstract}

A saúde bucal geral e a qualidade de vida foram piores entre os diabéticos em comparação com os não diabéticos. A saúde periodontal era pior e precisava de mais atenção entre os diabéticos.

A periodontite leve/moderada e grave esteve significativamente associada à pior QVRS. A periodontite leve/moderada impactou na incapacidade psicológica e a grave impactou na dor física, desconforto psicológico, incapacidade física e psicológica, desconforto psicológico.

A periodontite exerce influência sobre a QVRS em indivíduos com doença renal terminal, com uma condição mais grave afetando diferentes variáveis.

A ocorrência combinada de cárie dentária e

Mostrou que em indivíduos expostos simultaneamente à cárie e periodontite, a ocorrência do impacto na qualidade et al. 2018 / Estudo exposição combinada à cárie dentária transversal/ e periodontite e o impacto na qualidade de vida relacionada à saúde $306(18-80$ anos $) \quad$ bucal, por meio de exame clínico e questionário OHIP-14.

de vida foi $63 \%$ maior do que naqueles sem essas doenças.

Perda dentária, cárie dentária, pior estado periodontal e tabagismo estavam diretamente relacionados a uma pior QVRS. Renda e tabagismo influenciam indiretamente a qualidade de vida associada a saúde bucal devido a má condição periodontal. periodontite está associada a um impacto significativo na qualidade da saúde bucal quando comparada com a ausência dessas doenças bucais.

Há impacto negativo da doença periodontal na QVRS em pessoas com hipertensão arterial. Há associações de características sociodemográficas mediadas pela condição periodontal e tabagismo com QVRS.
Os escores de mobilidade dentária e perda de inserção clínica mostraram-se significativamente associados à qualidade de vida relacionada à saúde
A população geriátrica, especialmente aqueles em lares de idosos, é um grupo de necessidades especiais por causa 
Estudo transversal / versão pré-testada do Geriatric Oral Health Assessment Index (GOHAI$500(60-80$ anos $)$ $\mathrm{Hi})$ bucal, enquanto os dados do Índice Periodontal Comunitário mostraram uma correlação negativa. de sua incapacidade de acessar atendimento odontológico.

\begin{abstract}
Schmalz, G. et al. Avaliaram a qualidade de vida 2018 /Transversal/ relacionada à saúde bucal (QVRS), dependendo da saúde dentária e 146 (18 a 79 anos) periodontal de diferentes pacientes antes e após o transplante hepático, utilizando a versão alemã do Perfil de Impacto na Saúde Oral (OHIP G14).
\end{abstract}

A prevalência de periodontite foi comparável entre os grupos. A qualidade de vida relacionada a saúde bucal foi reduzida no pré e pós transplante sem associações aos seus estado oral.
A qualidade de vida relacionada a saúde bucal foi reduzida sem associações com seu estado oral, o que pode indicar uma influência de possíveis fatores relacionados à doença.

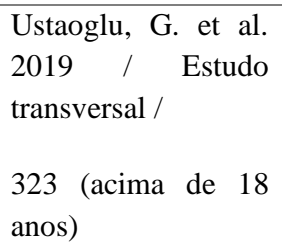

Investigaram o efeito da periodontite agressiva generalizada (PAG), periodontite crônica generalizada (PCG) e gengivite $(\mathrm{G})$ na qualidade de vida relacionada à saúde bucal, utilizando OHIP-14 e Short Questionários Form-36 (SF-36).
A PAG e os grupos PCG foram semelhantes entre si, exceto na limitação funcional e incapacidade social do questionário OHIP. O grupo PAG obteve pontuações mais altas que o grupo PCG em todas as subescalas do SF-36.

Os resultados do estudo indicam que diferentes formas de doença periodontal têm efeitos diferentes na qualidade de vida dos pacientes quando medidas pelo OHIP-14 e SF-36. Pacientes com PCG e PAG apresentaram pior qualidade de vida que pacientes com gengivite.

Wellapuli, N.

Ekanayake, L. 2016

/ Estudo transversal /

1400 (35-60 anos)
Determinaram o impacto da periodontite crônica na qualidade de vida relacionada à saúde bucal em adultos do Sri Lanka. Utilizou o questionário OHIP-14 para avaliação do impacto na qualidade de vida.
A prevalência, extensão e gravidade dos impactos orais aumentam com o aumento da gravidade da periodontite crônica. Os impactos mais comuns foram na variável da dor física. A periodontite crônica estava significativamente associada à prevalência de impactos orais.

Foi encontrado um efeito significativo entre o sextante anterior mandibular, componentes da ansiedade/medo dental e indicadores de QVRS. A região anterior da maxila foi significativamente associada ao desconforto da dor e funcionalidade.
A qualidade de vida relacionada à saúde bucal se deteriora com o aumento da gravidade da periodontite crônica.
Wright, C. D. et al. 2017 / Transversal analítico/ 1,339 (1893 anos)

\section{Avaliaram a qualidade de} relacionada à saúde bucal (QVRS) por meio do Questionário de Medo da Dor-9 e Perfil de Impacto na Saúde Oral-14.
A prevenção odontológica/medo antecipado e a excitação fisiológica influenciam a QVRS em indivíduos que apresentam indicadores de doença periodontal em sextantes que podem ser visíveis e suscetíveis a maior dor e impacto psicossocial.

\begin{abstract}
Yaacob, M. et al. Avaliaram o nível de conhecimento 2019 / Estudo das doenças periodontais e sua transversal /

80 (40-60 anos) correlação com a percepção de QVRS, utilizando a versão malaia do OHQoL-UK.
\end{abstract}

A maioria do grupo de alto conhecimento não percebeu nenhum efeito da doença periodontal na qualidade de vida. Apesar disso, foi encontrada correlação significativa, porém com menor força, entre $\mathrm{o}$ conhecimento dos pacientes e os aspectos sociais da OHQoL.

\footnotetext{
Yadav, T. et al. 2019 Avaliaram o efeito acentuado das / Estudo condições periodontais na qualidade de vida relacionada à saúde bucal na Transversal / 450 população da Índia, utilizando o OHIP (30-60 anos)

14 para avaliar o impacto na qualidade de vida.
}

A prevalência de impacto oral foi de $59 \%$ e $81 \%$ em participantes com periodontite moderada e/ou periodontite grave, respectivamente. A extensão e a gravidade também foram consideradas
A qualidade de vida relacionada à saúde bucal piora com a extensão da gravidade da periodontite crônica.
Pacientes com periodontite crônica perceberam impacto negativo da doença na qualidade de vida associada a saúde bucal. 


\section{proporcionais à gravidade da}

periodontite.

Fonte: Autores.

O tipo de estudo mais prevalente foi o analítico transversal, sendo utilizado por 28 artigos. O tipo caso-controle foi utilizado por 3 artigos. Os demais tipos de estudos foram: transversal comparativo (2), derivada de dois estudos de coortes (2) e análise secundária de dados transversais (1).

Todos os artigos utilizaram como forma de obtenção dos dados periodontais o exame clínico periodontal, com a exceção de um estudo, no qual utilizou um questionário de avaliação de capacidade mastigatória (Liang et al., 2020).

A ferramenta mais presente para a obtenção do impactado da qualidade de vida foi a OHIP-14, sendo utilizada por 27 estudos, seguido pelo questionário GOHAI (4), OHIP-49 (2), questionário de qualidade de vida da OMS (WHOQOL-BREF) (1), questionário desenvolvido pela Association for State and Territorial Dental Directors of the United States (1), questionário PSQI (1), Corah's Dental Escala de Ansiedade (DAS) (1), questionário SF - 36( 1), DFS e FPQ-9 (1), OHQoL-UK (1) e por fim, uma ferramenta projetada em um estudo piloto (1).

Os resultados mostram que a doença periodontal causa um impacto negativo na qualidade de vida relacionada à saúde oral (Al-Kattan et al., 2019; Ansari Moghadam et al., 2015; El Tantawi \& AlAgl, 2017; Eltas et al., 2016; Fuller et al.; Geevarghese et al., 2017; Goel \& Baral, 2017; Göktürk \& Uçan Yarkaç, 2018; He et al., 2018; Holde et al., 2018; Irani et al., 2015; Karaaslan \& Dikilitas, 2019; Levin, Zini, Levine, Weiss, Lev, Taub, et al., 2018; Levin, Zini, Levine, Weiss, Lev, Hai, et al., 2018; Liang et al., 2020; Llanos et al., 2018; Maia et al., 2018; Masood et al., 2019; Meusel et al., 2015; Musurlieva \& Stoykova, 2015; Nayak et al., 2017; Oliveira et al., 2020; Passos-Soares et al., 2018; Rebelo et al., 2016; Rekhi et al., 2016; Schmalz et al., 2018; Ustaoglu et al., 2019; Wellapuli \& Ekanayake, 2016; Wright et al., 2017; Yaacob et al., 2019; Yadav et al., 2019). Além disso, a doença foi associada a uma diminuição na qualidade do sono (Karaaslan \& Dikilitas, 2019), e quanto mais severa era a destruição periodontal, pior era a qualidade de vida(Fuller et al.; Göktürk \& Uçan Yarkaç, 2018; He et al., 2018; Karaaslan \& Dikilitas, 2019; Levin, Zini, Levine, Weiss, Lev, Hai, et al., 2018; Llanos et al., 2018; Meusel et al., 2015; Rebelo et al., 2016; Ustaoglu et al., 2019; Wellapuli \& Ekanayake, 2016; Yadav et al., 2019). Alguns estudos encontraram relações significativas entre a quantidade de dentes remanescentes e mobilidade dentária em relação à qualidade de vida(Kato et al., 2018; Lu et al., 2015; Marya et al., 2020; Rekhi et al., 2016).

Os estudos também encontraram resultados com alto impacto na qualidade de vida nos seguintes grupos: fumantes, ausência de seguro de saúde, obesos e diabéticos, grávidas, ansiosos, idosos, pacientes com doença renal terminal, cárie, hipertensão arterial (El Tantawi \& AlAgl, 2017; Freitas, 2015; Geevarghese et al., 2017; Holde et al., 2018; Irani et al., 2015; Levin, Zini, Levine, Weiss, Lev, Taub, et al., 2018; Levin, Zini, Levine, Weiss, Lev, Hai, et al., 2018; Liang et al., 2020; Nayak et al., 2017; Oliveira et al., 2020; Passos-Soares et al., 2018; Rebelo et al., 2016; Rekhi et al., 2016; Wright et al., 2017)

Entretanto, alguns estudos não encontraram relações significativas entre a doença e a qualidade de vida ou obtiveram baixo impacto (Andrade et al., 2019; Freitas, 2015; Kato et al., 2018; Lu et al., 2015).

\section{Discussão}

A maior parte dos estudos incluídos nesta revisão de literatura utilizou o delineamento do tipo transversal como método, ou seja, todas as medições foram feitas em uma única ocasião ou durante um curto período de tempo. Nenhuma abordagem é melhor do que as demais, e cada questão de pesquisa requer uma escolha racional sobre que desenho seria o mais eficiente na obtenção de uma resposta adequada (Hulley, Cummings, \& Brower, 2015).

Uma vez que estudos transversais medem apenas a prevalência, e não a incidência, é preciso cautela ao buscar inferir, 
por meio sobre causalidade, prognóstico ou história natural de uma doença, por meio destes estudos. Um fator que está associado a uma maior prevalência de uma doença pode ser a causa da mesma, mas também pode estar associado a uma duração mais prolongada (Hulley et al., 2015). Este problema parece ter acontecido em um estudo com adolescentes (Andrade et al., 2019), no qual se acredita que a ausência do impacto negativo seja devido a uma fase inicial da periodontite, necessitando assim de uma abordagem longitudinal.

Apenas um estudo utilizou questionário de avaliação de capacidade mastigatória como forma de avaliação clínica (Liang et al., 2020), desta forma, a presença da doença periodontal dependerá da auto percepção dos entrevistados, e estes, tratam-se de idosos, os quais muitas vezes não compreendem sua real necessidade de saúde bucal, as doenças bucais se tornam menos importantes, tornando-se menos perceptíveis. Além disso, esta forma de avaliação impossibilita medir a gravidade da doença, gerando viés no resultado da pesquisa. Dessa forma, este foi um dos problemas encontrados na grande maioria dos estudos incluídos nesta revisão.

Apesar das limitações dos artigos incluídos nesta revisão foi visto que a infecção periodontal pode aumentar significativamente o risco em relação a certas doenças sistêmicas ou alterar a evolução natural de condições sistêmicas. Entre as doenças sistêmicas, podemos citar o diabetes melito, a qual apresenta prevalência e gravidade aumentadas em relação à doença periodontal, especialmente aqueles com mau controle metabólico e é considerada a "sexta complicação do diabetes". Assim como o diabetes melito, o fumo também é um fator de risco bem estabelecida para a periodontite e afeta negativamente o resultado do tratamento, o que pode justificar o aumento do impacto da qualidade de vida (Carranza, Newman, Takei, \& Klokkevold, 2012).

O impacto na qualidade de vida foi maior em grávidas do que nas mulheres não grávidas. Uma possível razão para isto se deve a tendência na redução da frequência de visitas ou adiamento completo do atendimento odontológico durante a gravidez por medo de colocar a segurança da criança em risco (Geevarghese et al., 2017). A redução da frequência e até mesmo a ausência de acompanhamento odontológico também acontece em pacientes ansiosos e que tem medo da dor (Wright et al., 2017), fazendo com que esses grupos de pessoas sejam mais vulneráveis em relação às doenças bucais devido à falta de cobertura e acesso a serviços odontológicos. Algo parecido acontece com as pessoas sem seguro de saúde (El Tantawi \& AlAgl, 2017), pois apresentaram um impacto negativo maior na qualidade de vida e isso pode ser explicado pela falta de acesso a serviços especializados, promovendo um impacto na prevalência e severidade da doença.

Sabendo disso, tornam-se necessárias estratégias para difundir informações sobre atendimento odontológico e desmistificar informações que levam a evasão das grávidas antes e durante o acompanhamento odontológico, favorecendo uma maior busca pelo pré-natal odontológico, proporcionando uma maior segurança e vínculo das pacientes com a equipe. Essa segurança também é necessária para os pacientes ansiosos, os quais tem medo do atendimento odontológico, sendo necessárias estratégias para minimizar os estresses durante o atendimento, um exemplo seria o treinamento dos profissionais em relação a técnicas menos invasivas de anestésicos, proporcionando um maior conforto ao paciente e aceitação ao tratamento.

A periodontite foi associada também a uma pior qualidade de vida em pacientes hipertensos, entretanto, não houve um grupo controle para comparar essa associação com pacientes saudáveis (Rebelo et al., 2016). Estudos apontam que pacientes com doença periodontal enfrentam maior risco de desenvolver hipertensão quando comparados a indivíduos com periodonto saudável (Zhao et al., 2019), desta forma, a doença periodontal tem um impacto negativo na saúde sistêmica e consequentemente na qualidade de vida.

A associação da cárie dentária com a periodontite apresentou impacto negativo na qualidade de vida relacionada à saúde bucal (Passos-Soares et al., 2018), ou seja, além das doenças sistêmicas, a coexistência de doenças bucais, como a cárie, potencializa o impacto negativo na qualidade de vida. Isto pode ser explicado pelo fato de que a cárie tem uma característica sintomática muito presente, principalmente quando causa uma pulpite aguda, sendo uma das principais queixas de dor no 
atendimento odontológico.

Tratando-se do grupo de pacientes com insuficiência renal, a doença renal crônica pode predispor e/ou agravar a doença periodontal, da mesma forma que, em sentido inverso, a doença periodontal pode ser fator de comorbidade e fonte oculta de inflamação, podendo causar e/ou predispor ao dano renal agravando a doença, inclusive risco de dano ao enxerto renal (Almeida et al., 2011). Desta forma, os pacientes devem ser mantidos sobre regular acompanhamento odontológico e controle de higienização oral a fim de manter a saúde bucal.

Contudo, é necessária uma melhor compreensão e percepção em relação ao impacto da doença periodontal na qualidade de vida, pois dessa forma é possível identificar necessidades e preocupações dos pacientes e entender melhor os fatores associados ao problema exposto, além de permitir e facilitar a produção de estratégias que busquem minimizar os impactos negativos produzidos pela doença e garantir o planejamento e tratamento adequados para os pacientes.

\section{Conclusão}

As doenças periodontais interferem negativamente na qualidade de vida das pessoas afetadas e há evidências de quanto maior a severidade da doença, maior é o seu impacto na qualidade de vida. Contudo, são necessários mais estudos com maior nível de evidência científica, como estudos randomizados controlados e estudos do tipo longitudinal para fortalecer estes achados. Além disso, recomenda-se que o tratamento seja feito por um período maior de tempo para verificar os efeitos da terapia em longo prazo.

\section{Agradecimentos}

Agradeço pelo apoio da Coordenação de Aperfeiçoamento de Pessoal de Nível Superior - Brasil (CAPES) - Código Financiador 001.

\section{Referências}

Al-Kattan, R., Al Shibani, N., Al Zahrani, H., Al Enazi, G. \& Al Nafa, D. (2019). Influence of Periodontal Disease on QoL of Periodontal Patients in Riyadh. Journal of Advanced Oral Research, 10(2), 85-90. doi:10.1177/2320206819848248

Almeida, D., Sardenberg, C., Granjeiro, J., Machado, W., Tostes, F. \& Barboza, E. (2011). A relação bidirecional entre doença periodontal e doença renal crônica: da progressão da doença renal crônica à terapia renal substitutiva de diálise. Revista Periodontia, 21, 71-77.

Andrade, A. L., Callado, A. T., Sousa, A. M., Silva, T. C., Cimões, R. \& Vajgel, B. F. (2019). Association between quality of life and periodontal disease in adolescents. Adolescencia e Saude, 16(3), 59-69.

Ansari Moghadam, S., Abdollahi, Z., Risbaf Fakour, S., Ansari Moghaddam, A., Kiany, F. \& Damani, N. (2015). The Relationship Between Periodontal Disease and Public Health: A Population-Based Study. Glob J Health Sci, 8(7), 110-115. doi:10.5539/gjhs.v8n7p110

Carranza, F. A.; Newman, M. G.; Takei, H. H. \& Klokkevold, P. R. (2012). Periodontia Clínica (11 ed.). Saunders Elsevier.

Dantas, F. T., Martins, S. H. L., Dantas, A. T. d. M. \& Gnoatto, N. (2016). Stress and periodontal disease: a literature review. Revista de Periodontia, 26(3), 1928 .

El Tantawi, M. \& AlAgl, A. (2017). Disability and the impact of need for periodontal care on quality of life: A cross-sectional study. J Int Med Res, 45(6), 19491960. doi:10.1177/0300060517715376

Eltas, A., Uslu, M. O. \& Eltas, S. D. (2016). Association of Oral Health-related Quality of Life with Periodontal Status and Treatment Needs. Oral Health \& Preventive Dentistry, 14(4), 339-347. doi:10.3290/j.ohpd.a35613

Freitas, A. R. d. (2015). Periodontal conditions and oral hygiene, quality of life and satisfaction with life in obese diabetic and nondiabetic patients undergoing bariatric surgery. 140-140.

Fuller, J., Donos, N., Suvan, J., Tsakos, G. \& Nibali, L. Association of oral health-related quality of life measures with aggressive and chronic periodontitis. Journal of Periodontal Research. doi:10.1111/jre.12745

Geevarghese, A., Baskaradoss, J. K. \& Sarma, P. S. (2017). Oral Health-Related Quality of Life and Periodontal Status of Pregnant Women. Matern Child Health J, 21(8), 1634-1642. doi:10.1007/s10995-016-2255-y 
Goel, K. \& Baral, D. (2017). A Comparison of Impact of Chronic Periodontal Diseases and Nonsurgical Periodontal Therapy on Oral Health-Related Quality of Life. Int J Dent. doi:10.1155/2017/9352562

Göktürk, Ö. \& Uçan Yarkaç, F. (2018). Assessment of oral health-related quality of life among elderly patients with periodontal disease. Turk Geriatri Dergisi, 21(3), 313-323. doi:10.31086/tjgeri.2018344044

He, S., Wei, S., Wang, J. \& Ji, P. (2018). Chronic periodontitis and oral health-related quality of life in Chinese adults: A population-based, cross-sectional study. J Periodontol, 89(3), 275-284. doi:10.1002/jper.16-0752

Holde, G. E., Baker, S. R. \& Jonsson, B. (2018). Periodontitis and quality of life: What is the role of socioeconomic status, sense of coherence, dental service use and oral health practices? An exploratory theory-guided analysis on a Norwegian population. J Clin Periodontol, 45(7), 768-779. doi:10.1111/jcpe.12906

Hulley, S. B.; Cummings, S. R. \& Brower, W. S. (2015). Delineando a pesquisa clínica (4 ed.). Artmed.

Irani, F. C., Wassall, R. R. \& Preshaw, P. M. (2015). Impact of periodontal status on oral health-related quality of life in patients with and without type 2 diabetes. Journal of Dentistry, 43(5), 506-511. doi:10.1016/j.jdent.2015.03.001

Karaaslan, F. \& Dikilitas, A. (2019). The association between stage-grade of periodontitis and sleep quality and oral health-related quality of life. J Periodontol, 90(10), 1133-1141. doi:10.1002/jper.19-0034

Kato, T., Abrahamsson, I., Wide, U. \& Hakeberg, M. (2018). Periodontal disease among older people and its impact on oral health-related quality of life. Gerodontology, 35(4), 382-390. doi:10.1111/ger.12363

Kreve, S. \& Anzolin, D. (2016). Impacto da saúde bucal na qualidade de vida do idoso. Revista Kairós, 19(22). doi:10.23925/2176901X.2016v19iEspecial22p45-59

Levin, L., Zini, A., Levine, J., Weiss, M., Lev, R., Taub, D. C. \& Almoznino, G. (2018). Demographic profile, Oral Health Impact Profile and Dental Anxiety Scale in patients with chronic periodontitis: a case-control study. International Dental Journal, 68(4), 269-278. doi:10.1111/idj.12381

Levin, L., Zini, A., Levine, J., Weiss, M., Lev, R. A., Hai, A. \& Almoznino, G. (2018). Dental anxiety and oral health-related quality of life in aggressive periodontitis patients. Clin Oral Investig, 22(3), 1411-1422. doi:10.1007/s00784-017-2234-8

Liang, Y. H., Chou, C., Chen, Y. J., Chou, Y. F., Lin, C. Y. \& Wang, T. F. (2020). Impact of periodontal disease and chewing ability on the quality of life of the elderly in an affluent community. Journal of the Formosan Medical Association. doi:10.1016/j.jfma.2020.01.002

Llanos, A. H., Silva, C. G. B., Ichimura, K. T., Rebeis, E. S., Giudicissi, M., Romano, M. M. \& Saraiva, L. (2018). Impact of aggressive periodontitis and chronic periodontitis on oral health-related quality of life. Braz Oral Res, 32. doi:10.1590/1807-3107bor-2018.vol32.0006

Lopes, M. W. F., Gusmão, E. S., Alves, R. d. V. \& Cimões, R. (2011). Impacto das doenças periodontais na qualidade de vida. RGO, 59(supl.1), 39-44.

Lu, H. X., Xu, W., Wong, M. C., Wei, T. Y. \& Feng, X. P. (2015). Impact of periodontal conditions on the quality of life of pregnant women: a cross-sectional study. Health Qual Life Outcomes, 13. doi:10.1186/s12955-015-0267-8

Maia, C. D. R., Mendes, F. M. \& Normando, D. (2018). The impact of oral health on quality of life of urban and riverine populations of the Amazon: A multilevel analysis. Plos One, 13(11). doi:10.1371/journal.pone.0208096

Marya, C., Grover, H., Tandon, S., Taneja, P., Gupta, A. \& Marya, V. (2020). Gender-wise comparison of oral health-related quality of life and its relationship with periodontal status among the Indian elderly. Journal of Indian Society of Periodontology, 24(1), 72-79. doi:10.4103/jisp.jisp_156_19

Masood, M., Younis, L. T., Masood, Y., Bakri, N. N. \& Christian, B. (2019). Relationship of periodontal disease and domains of oral health-related quality of life. J Clin Periodontol, 46(2), 170-180. doi:10.1111/jcpe.13072

Mendez, M. \& Gomes, S. C. (2013). Qualidade de vida relacionada à saúde bucal: perspectivas para a periodontia. Periodontia, 23(4), 38-44.

Meusel, D. R., Ramacciato, J. C., Motta, R. H., Brito Junior, R. B. \& Florio, F. M. (2015). Impact of the severity of chronic periodontal disease on quality of life. J Oral Sci, 57(2), 87-94. doi:10.2334/josnusd.57.87

Musurlieva, N. \& Stoykova, M. (2015). Evaluation of the impact of chronic periodontitis on individual's quality of life by a self-developed tool. Biotechnology and Biotechnological Equipment, 29(5), 991-995. doi:10.1080/13102818.2015.1058189

Nayak, S. U., Kamath, D. G. \& Pai, K. K. (2017). Periodontal health and its impact on quality of life among type II diabetes mellitus patients. World Journal of Dentistry, 8(2), 86-89. doi:10.5005/jp-journals-10015-1418

Neves, P. C. B., Cortellazzi, K. L., Ambrosano, G. M. B., Pereira, A. C., Meneghim, M. d. C. \& Mialhe, F. L. (2013). Variáveis sociodemográficas e psicocomportamentais associadas à gengivite e à pobre higiene bucal em pacientes de uma unidade de saúde da família. Periodontia, 23(2), 16-24.

Oliveira, L. M., Sari, D., Schoffer, C., Santi, S. S., Antoniazzi, R. P. \& Zanatta, F. B. (2020). Periodontitis is associated with oral health-related quality of life in individuals with end-stage renal disease. Journal of Clinical Periodontology, 47(3), 319-329. doi:10.1111/jcpe.13233

Passos-Soares, J. D., Santos, L. P. D., da Cruz, S. S., Trindade, S. C., Cerqueira, E. D. M., Santos, K. O. B. \& Gomes, I. S. (2018). The impact of caries in combination with periodontitis on oral health-related quality of life in Bahia, Brazil. Journal of Periodontology, 89(12), 1407-1417. doi:10.1002/jper.18-0047

Rebelo, M. A., de Castro, P. H., Rebelo Vieira, J. M., Robinson, P. G. \& Vettore, M. V. (2016). Low Social Position, Periodontal Disease, and Poor Oral HealthRelated Quality of Life in Adults with Systemic Arterial Hypertension. J Periodontol, 87(12), 1379-1387. doi:10.1902/jop.2016.160204

Rekhi, A., Marya, C. M., Oberoi, S. S., Nagpal, R., Dhingra, C. \& Kataria, S. (2016). Periodontal status and oral health-related quality of life in elderly residents of aged care homes in Delhi. Geriatr Gerontol Int, 16(4), 474-480. doi:10.1111/ggi.12494 
Research, Society and Development, v. 10, n. 3, e17110313160, 2021

(CC BY 4.0) | ISSN 2525-3409 | DOI: http://dx.doi.org/10.33448/rsd-v10i3.13160

Schmalz, G., Meisel, A., Kollmar, O., Kauffels, A., Slotta, J. E., Kottmann, T. \& Ziebolz, D. (2018). Oral health-related quality of life depending on dental and periodontal health in different patients before and after liver transplantation. Clin Oral Investig, 22(5), 2039-2045. doi:10.1007/s00784-017-2298-5

Ustaoglu, G., Goller Bulut, D., Gumus, K. C. \& Ankarali, H. (2019). Evaluation of the effects of different forms of periodontal diseases on quality of life with OHIP-14 and SF-36 questionnaires: A cross-sectional study. Int J Dent Hyg, 17(4), 343-349. doi:10.1111/idh.12409

Wellapuli, N. \& Ekanayake, L. (2016). Association between chronic periodontitis and oral health-related quality of life in Sri Lankan adults. Int Dent J, 66(6), 337-343. doi:10.1111/idj.12255

The World Health Organization Quality of Life assessment (WHOQOL) (1995): position paper from the World Health Organization. Soc Sci Med, 41(10), 14031409. doi:10.1016/0277-9536(95)00112-k

Wright, C. D., McNeil, D. W., Edwards, C. B., Crout, R. J., Neiswanger, K., Shaffer, J. R. \& Marazita, M. L. (2017). Periodontal Status and Quality of Life: Impact of Fear of Pain and Dental Fear. Pain Res Manag. 2017. 5491923. doi:10.1155/2017/5491923

Yaacob, M., Han, T. M., Wahab, S. M. A., Sham, S. A. M. \& Abllah, Z. (2019). Chronic periodontitis patients: Their knowledge and its correlation with oral health related quality of life C3 - Materials Today: Proceedings. Bio-CAM 2017, 16, 2302-2308.

Yadav, T., Chopra, P. \& Kapoor, S. (2019). Association between chronic periodontitis and oral health-related quality of life in Indian adults. Journal of International Oral Health, 11(5), 280-286. doi:10.4103/jioh.jioh_50_19

Zhao, M. J., Qiao, Y. X., Wu, L., Huang, Q., Li, B. H. \& Zeng, X. T. (2019). Periodontal Disease Is Associated With Increased Risk of Hypertension: A CrossSectional Study. Front Physiol, 10, 440. doi:10.3389/fphys.2019.00440 